\title{
Learning Bilingual Collocations by Word-Level Sorting
}

\author{
Masahiko Haruno Satoru Ikehara Takefumi Yamazaki \\ N'T'T' Communication Science Labs. \\ 1-2356 Take Yokosuka-Shi \\ Kanagawa 238-03, Japan \\ haruno@nttkb.ntt.jp ikehara@nttkb.ntt.jp yamazaki@nttkb.ntt.jp
}

\begin{abstract}
This paper proposes a new method for learning; bilingual collocations from sentence-aligned parallel corpora. Our method comprises two steps: (1) extracting useful word chunks (n-grams) by word-level sorting and (2) constructing bilingual collocations by combining, the word-chunks acquired in stage (1). We apply the method to a very chat lenging text pair: a stock market, bulletin in Japanese and its abstract in linglish. Domain specific collocations are well captured even if they were not contained in the dictionaries of economic: terms.
\end{abstract}

\section{Introduction}

In the field of machine translation, there is a growing interest in corpus-based approaches (Sato and Nagao, 1990; Dagan and (hurch, 1994; Mat-sumoto ot al., 1993; Kumano and Ilirakawa, 1994; Smadja et al., 1996). 'The main motivation behind this is to well handle domain specific oxpressions. lach application domain has varjous kinds of collocations ranging from word-level to sentence-level. The correct use of these collocations greatly influences the quality of output texts. Because such detailed collocations are difficult to hand-compile, the autonatic extraction of bilin gual collocations is needed.

A number of studies have attempted to extract bilingual collocations from parallel corporat 'These studies can be classified into two directions. One is based on the full parsing techniques. (Matsumoto et al., 1993) proposed a medhol to find ont phrase-level correspondences, while resolving syntactic ambiguities at the same time. 'Their methods determine phraso correspondencess by using the phrase structures of the two languages and existing bilingual dictionaries. Unfortunately these approaches are promising only for the compara dively short sentences that can be analyzed by in C KY type parser.
'The other direction for extracting bilingual collocations involves statistics. (lung, 1995) acquired bilingual word correspondences without sentence aligmment. Although these methods are robust and assume no information sourec, their outputs are just word-word correspondences. (Kupiec, 1993; Kumano and Ilirakawa, 1994) extracted noun phrase (NP) correspondences from aligned parallel corpora. In (Kupiec, 1993), NT's in Finglish and french texts are first extracted by a $\mathrm{Nl}^{\prime}$ recognizer. 'Their correspondence prol,abilities are then gradually refined by using an liM-like iteration algorithm. (Kmmano and IIrakawa, 1994) first extracted Japanese NPs in the sane way, and combined statistics with a bilingrual dictionary for M'I' to find out NP' corresponances. Nlthough their approaches attained high accuracy for the task considered, the most crucial knowledge for M'l' is more complex correspondences such as NP-VP correspondences and sentence-level correspondences. It secms difficult to extend these statistical methods to a broader range of collocations because they are specialized to N l's or single words.

(Smadja ef al., 1996) proposed a general method to extract a broader range of collocations. 'They first extract linglish collocations using the Xtract system (Smadja, 1993), and then look for Fench comberparts. Their search strategy is an iterative combination of two elements. 'This is based on the intuitive idea that "if a set of words constitutes a collocation, its subset will also be correlated". Althomgh this idea is correct, the itcrative combination strategy generates a number of useless expressions. In fact, Xtract cmploys a robust linglish parser to lilter out the wrong collocations which form more than half the candidates. In other languages such as Japanese, parser-based pruning cannot be used. Another drawlack of their approach is that only the longest n-gratn is adopted. 'That is, when 'J apan-US' anto trade talks' is adopted as a collocation, 'Japan-US' cannot be recognized as a collocation though it is independently used very often.

In this paper, we propose an altemative method based on word-level sorting. One method com- 
prises two steps: (1) extracting useful word chunks (n-grams) by word-level sorting and (2) constructing bilingual collocations by combining the wordchunks acquired at stage (1). Given sentencealigned texts in two languages(Haruno and Yamazaki, 1996), the first step detects useful word chunks by sorting and counting all uninterrupted word sequences in sentences. In this phase, we developed a new technique for extracting only useful chunks. The second step of the method evaluates the statistical similarity of the word chunks appearing in the corresponding sentences. Most of the fixed (uninterrupted) collocations are directly extracted from the word chunks. More flexible (interrupted) collocations are acquired level by level by iteratively combining the chunks. The proposed method, which uses effective word-level sorting, not only extracts fixed collocations with high precision, but also avoids the combinatorial explosion involved in searching flexible collocations. In addition, our method is robust and suitable for real-world applications because it only assumes part-of-speech taggers for both languages. Even if the part-of-speech taggers make errors in word segmentation, the errors can be recovered in the word chunk extraction stage.

\section{Two Types of Japanese-English Collocations}

In this section, we briefly classify the types of Japanese-English collocations by using the material in Table 1 as an example. These texts were derived from a stock market bulletin written in Japanese and its abstract written in English, which were distributed electrically via a computer network.

In Table 1, (東京外為 / Tokyo Forex), (日米自動 車問題 / auto talks between Japan and the U.S.) and (控之て/ahead of) are Japanese-English collocations whose elements constitute uninterrupted word sequences. We call hereafter this type of collocation fixed collocation. Although fixed collocation seems trivial, more than half of all useful collocations belong to this class. Thus, it is important to extract fixed collocations with high precision. In contrast, (ドルは〜で取り引き終充 ₹/The U.S. currency was quoted at $\sim$ ) and $\left(F^{*}\right.$ ルは〜で取り引を終えた / The dollar stood 〜 $)^{1}$ are constructed from interrupted word sequences. We will call this type of collocation flexible collocation. From the viewpoint of machine learning, flexible collocations are much more difficult to learn because they involve the combination of elements. The points when extracting flexible collocations is how the number of combination (candidates) can be reduced.

Our learning method is twofold according to the collocation types. First, useful uninterrupted

\footnotetext{
$1 \sim$ represents any sequence of words.
}

word chunks are extracted by the word-level sorting method. To find out fixed collocations, we evaluate stochastic similarity of the chunks. Next, we iteratively combin the chunks to extract flexible collocations.

\section{Extracting Useful Chunks by Word-Level Sorting}

\subsection{Previous Research}

With the availability of large corpora and memory devices, there is once again growing interest in extracting $n$-grams with large values of $n$. (Nagao and Mori, 1994) introduced an efficient method for calculating an arbitrary number of n-grams from large corpora. When the length of a text is $l$ bytes, it occupies $l$ consecutive bytes in memory as depicted in Figure 1. First, another table of size $l$ is prepared, each field of which represents a pointer to a substring. A substring pointed to by the $(i-1)$ th entry of the table constitutes a string existing from the $i$ th character to the end of the text string. Next, to extract common substrings, the pointer table is sorted in alphabetic order. Two adjacent words in the pointer table are compared and the lengths of coincident prefix parts are counted(Gonnet et al., 1992).

For example, when 'auto talks between Japan and the U.S.' and 'auto talks between Japan and China' are two adjacent words, the number of coincidences is 29 as in 'auto talks between. Japan and '. The n-gram frequency table is constructed by counting the number of pointers which represent the same prefix parts. Although the method is efficient for large corpora, it involves large volume of fractional and unnecessary expressions. The reason for this is that the method does not consider the inter-relationships between the extracted strings. That is, the method generates redundant substrings which are subsumed by longer strings.

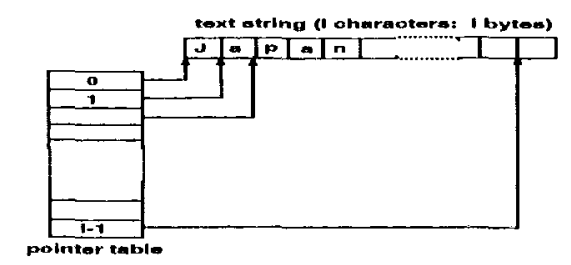

Figure 1: Nagao's Approach

To settle this problem, (Ikehara et al., 1996) proposed a method to extract only useful strings. Basically, his methods is based on the longestmatch principle. When the method extracts a longest n-gram as a chunk, strings subsumed by the chunk are derived only if the shorter string often appears independently to the longest chunk. If 'auto talks between Japan and the U.S.' is extracted as a chunk, 'Japan and the U.S.' is also 
1. 東京外為 17 時・日、小反発- -26 銭高 84 円 $21-24$ 銭。 Tokyo Forex 5 PM: Dollar at 84.21-84.24 yen

2. 前週末比 26 銭门高ドル安の 1 ドル=84円 $21-24$ 銭で大方の取引を柊えた。 The dollar stood 0.26 yen lower at 84.21-84.24 at 5 p.m.

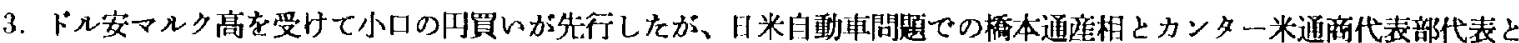

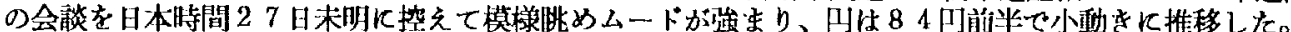

Forex market trading was extremely quict ahead of further auto talks between Japan and the U.S., slated for early dawn Thesday.

4. ドルは対マルクで反洛し 1 ドルニ1.3863-66マルクではば收引を格えた。 The U.S. currency was quoted at 1.361-1.3863 German marks at 5:15 p.m.

'Table 1: Sample of Target Texts

extracted because 'Japan and the U.S.' is used so often independently as in 'Japan and the U.S. agreed ...'. However, 'Japan and the' is not extracted because it always appears in the context of 'Japan and the U.S.'. The method strongly suppresses fractional and unnecessary expressions. More than $75 \%$ of the strings extracted by $\mathrm{Na}-$ gao's method are removed with the new method.

\subsection{Word-Level Sorting Method}

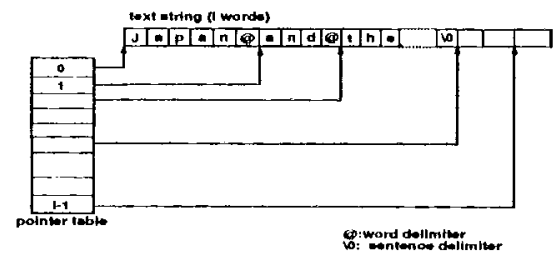

Figure 2: Word-Ievel Sorting Approach

The research described in the previous section deals with character-based n-grams, which generate excessive numbers of expressions and requires large memory for the pointer table. Thus, from a practical point of view, word-based n-grams are preferable in order to further suppress fractional expressions and pointer table use. In this paper, we extend Ikehara's method to handle word-based n grams. First, both Japanese and English texts are part-of-spech (POS) tagged ${ }^{2}$ and stored in memory as in Figure 2. POS tagging is required for two main reasons: (1) There are no explicit word delimiters in Japanese and (2) By using POS information, useless expressions can be removed.

In Figure 2, '@' and ' $\backslash 0$ ' represent the explicit word delimiter and the explicit sentence delimiter, respectively. Compared to previous research, this data structure has the following advantages.

\footnotetext{
${ }^{2}$ We use in this phase the JUMAN morphological analyzing system (Kurohashi et al., 1994) for tagging Japanese texts and Brill's transformation-based tagger (Brill, 1994) for tagging Finglish text.s. We wonld like to thank all people concerned for providing us with the tools.
}

1. Only heads of each word are recorded in the pointer table. As depicted in Figure 2, this remarkably reduces memory use because the pointer table also contains other string characteristics as Figure 3 .

2. $\Lambda$ s depicted in Figure 2, only expressions within a sentence are considered by introducing the explicit sentence delimiter ' $\backslash 0$ '.

3. Only word-level coincidences are extracted by introducing the explicit word delimiter '@'. This removes strings arising from a partial match of different words. For example, the coincident string between 'Japan and China' and 'Japan and Costa Rica' is 'Japan and' in our method, while it is 'Japan and $C^{6}$ in previous methods.

\begin{tabular}{|c|c|c|c|}
\hline $\begin{array}{l}\text { sent } \\
\text { no. }\end{array}$ & aclopt & $\begin{array}{l}\text { Colnci } \\
\text { dence }\end{array}$ & string \\
\hline $\begin{array}{l}21 \\
10 x \\
1064 \\
-3 \\
2104 \\
1702 \\
110-4 \\
104\end{array}$ & & $\begin{array}{l}10 \\
103 \\
10 \\
10 \\
10 \\
16 \\
10 \\
10 \\
10\end{array}$ & 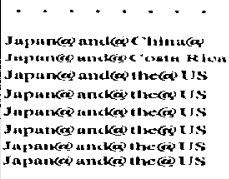 \\
\hline
\end{tabular}

Figure 3: Sorted Pointer Table

Next, the pointer table is sorted in alphabetic order as shown in Figure 3. In this table, sentno. and coincidence represent which sentence the string appeared in and how many characters are shared by the two adjacent strings, respectively. 'That is, coincidence delineates candidates for useful expressions. Note here that the coincidence between Japan@and@China... and Japan@and@Costa Rica. . is 10 as mentioned above.

Next, in order to remove useless subsumed strings, the pointer table is sorted according to sentno.. In this stage, adopt is filled with ' 1 ' or ' 0 ', each of which represents if or not if a string is subsumed by longer word chunks, respectively. Sorting by sentno. makes it much easier to check the subsumption of word chunks. When 
both 'Japan and the U.S.' and 'Japan and the' arise from a sentence, the latter is removed because the former subsumes the latter.

Finally, to determine which word-chunks to extract, the pointer table is sorted once again in alphabetic order. In this stage, we count how many times a string whose adopt is 1 appears in the corpus. By thresholding the frequency, only useful word chunks are extracted.

\section{Extracting Bilingual Collocations}

In this section, we will explain how JapaneseEnglish collocations are constructed from word chunks extracted in the previous stage. First, fixed collocations are induced in the following way. We use the contingency matrix to evaluate the similarity of word-chunk occurrences in both languages. Consider the contingency matrix, shown Table 2, for Japanese word chunk $c_{j p n}$ and English word chunk $c_{e n g}$. The contingency matrix shows: (a) the number of Japanese-English corresponding sentence pairs in which both $c_{j p n}$ and $c_{e n g}$ were found, (b) the number of Japanese-English corresponding sentence pairs in which just $c_{e n g}$ was found, (c) the number of Japanese-English corresponding sentence pairs in which just $c_{j p n}$ was found, (d) the number of Japanese-Hnglish corresponding sentence pairs in which neither chunk was found.

\begin{tabular}{|c|c|}
\hline & $c_{j p n}$ \\
\hline$c_{\text {eng }}$ & $\begin{array}{ll}a & b \\
c & d\end{array}$ \\
\hline
\end{tabular}

Table 2: Contingency Matrix

If $c_{j p n}$ and $c_{e n g}$ are good translations of one another, $a$ should be large, and $b$ and $c$ should be small. In contrast, if the two are not good translations of each other, a should be small, and $b$ and $c$ should be large. To make this argument more precise, we introduce mutual information as follows. Thresholding the mutual information extracts fixed collocations. Note that mutual information is reliable in this case because the frequency of cach word chunk is throsholded at the word chunk extraction stage.

$$
\log \frac{\operatorname{prob}\left(c_{j p n}, c_{e n g}\right)}{\operatorname{prob}\left(c_{j p n}\right) \operatorname{prob}\left(c_{e n g}\right)}=\log \frac{a(a+b+c+d)}{(a+b)(a+c)}
$$

Next, we summarize how flexible collocations arc extracted. The following is a scries of procedures to extract flexible collocations.

1. For any pair of chunks in a Japanese sentence, compute mutual information. Combine the two chunks of highest mutual information. Iteratively repeat this procedure and construct a tree level by level.
2. For any pair of chunks in an English sentence, repeat the operations done in the the Japanese sentence.

3. Perform node matching between trees of both languages by using mutual information of Japanese and English word chunks.

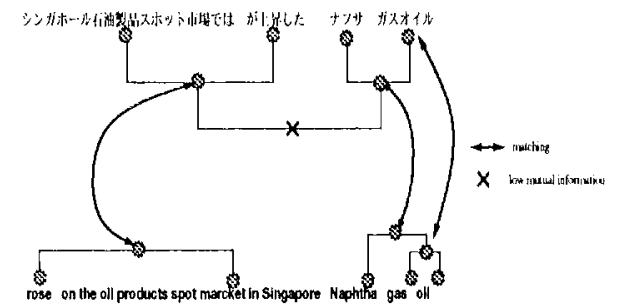

Figure 4: Constructing Flexible Collocations

The first two steps construct monolingual similarity trees of word chunks in sentences. The third step iteratively evaluates the bilingual similarity of word chunk combinations by using the above trees. Consider the example below, in which the underlined word chunks construct a flexible col-

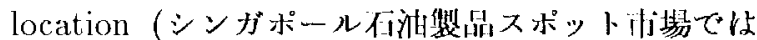

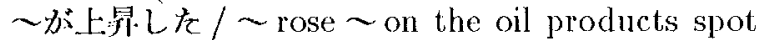
market in Singapore). First, two similarity trees are constructed as shown in Figure 4. Graph matching is then iteratively attempted by computing mutual information for groups of word chunks. In the present implementation, the system combines three word chunks at most. The technique we use is similar to the parsing-based methods for extracting bilingual collocation(Matsumoto et al., 1993). Our method replaces the parse trees with the similarity trees and thus avoids the combinatorial explosion inherent to the parsing-based methods.

$$
\begin{aligned}
& \text { Example: }
\end{aligned}
$$

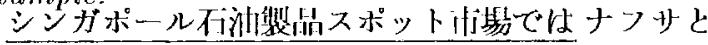

Naphtha and gas oil rose on the oil products spot market in Singapore

\section{Preliminary Evaluation and Discussion}

We performed a preliminary evaluation of the proposed mothod by using 10-days Japanese stock market bulletins and their Inglish abstracts, each containing 2000 sentences. The text was first automatically aligned and then hand-checked by a human supervisor. A sample passage is displayed in 'Table 1.

In this experiment, we considered only the word chunks that appeared nore than 4 times for fixed collocations and more than 6 times for flexible collocations. 'lable 4 illustrates the fixed collocations acquired by our method. Almost all collocations in Table 4 involve domain specilic jargon, which 


\begin{tabular}{|c|c|c|}
\hline No. & Japanese & English \\
\hline 1 & 東定外為 $-[1]=$ & Tokyo forex Dollar at $\sim$ yen \\
\hline 2 & 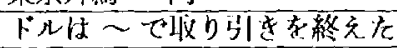 & The U.S currency was quoted at $\sim$ \\
\hline 3 & 一が管られ & $\sim$ were sold $\sim$ dropped as well \\
\hline 4 & 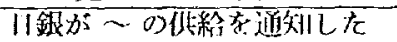 & Bank of Japan injected - \\
\hline 5 & 才ム口: 価友林二 & Omron Sumitomo Forestry - \\
\hline
\end{tabular}

Table 3: Samples of Flexible Collocations

\begin{tabular}{|c|c|c|c|c|c|}
\hline No: & Jupaucse & English & No. & Japanesse & linglish \\
\hline 1 & 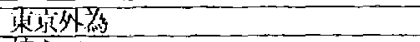 & Tokyo Forex & 38 & 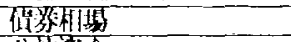 & bonds and bond futures \\
\hline 2 & 拱充 $\tau$ & ahead of & 39 & 公的实全 & public funds \\
\hline 3 & $\nabla \pi y$ & German mark & 40 & 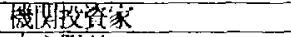 & inatitutional investors \\
\hline 4 & $\mathrm{JACO}$ & Japan Asbocinted Finance & 41 & $1(10 \mathrm{\beta}) 1$ & benchmark \\
\hline $\mathbf{b}$ & Fifi & in contrast & 42 & 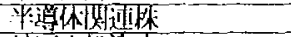 & semiconductor-related stocks \\
\hline 6 & 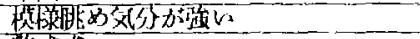 & remained sidelined watching & 43 & 外的人投象象 & foreign investors \\
\hline$?$ & 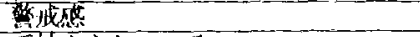 & fear & 44 & $\therefore 1 \% 314$ & high-tech stocks \\
\hline 8 & 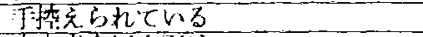 & awaiting & 45 & 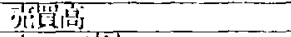 & turnover \\
\hline$\overline{9}$ & 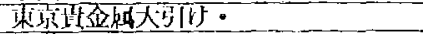 & Tokyo Gold futures Cla: & 46 & 小10要占 & small-lot selling \\
\hline 10 & 小野政 & slow & 47 & 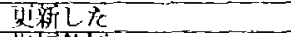 & record high \\
\hline 11. & d造可份 & wait-sind-sec mood & 48 & 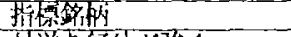 & berchinark \\
\hline 12 & 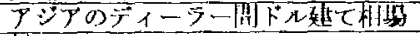 & Loco-London gold & 49 & 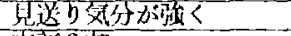 & low \\
\hline 13 & 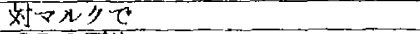 & against mark & 50 & 整徒 2 部 & Tokyo Stocks 2nd Sec \\
\hline 14 & 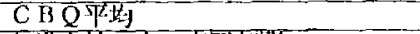 & Convertible bonds & b1 & 㜞湆 & were weak \\
\hline 15 & 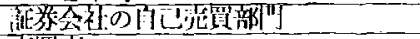 & deslers & 52 & 体人投纸家 & individual investors \\
\hline 16 & 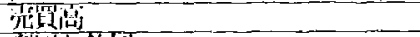 & trading volume & 53 & 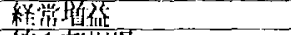 & pretax profit \\
\hline 17 & 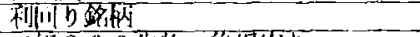 & high-yielders & 54 & 牙 1 部相是 & The firatsection of TSH \\
\hline 18 & 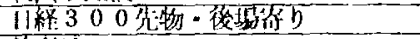 & Nikkei 300 futures Aft-opg: & 65 & 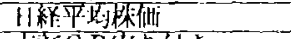 & the Nikkei stock average \\
\hline 19 & 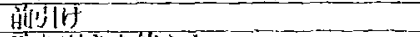 & mng-cls: & 56 & 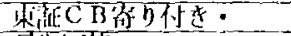 & Tokyo CBs Opg: \\
\hline 20 & 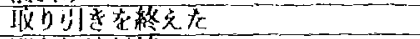 & contract onded & 67 & 东场国整 & long-term government bonds \\
\hline 21 & 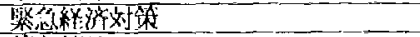 & economic stimulus package & 58 & 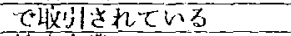 & were traded at \\
\hline 22 & 粕b fifits & losed $\mathrm{nt}$ & 69 & 期入金 & importers \\
\hline 23 & 先物-大亏方 & fintures cls: & 60 & 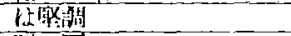 & advanced \\
\hline 24 & 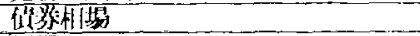 & bond merket & 61 & 罗以总L & covering \\
\hline 25 & 相穿 & convertible bonds & 62 & 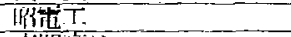 & Showa Denko \\
\hline 26 & 少物-移量的b & nikkei futures aft-opg: & 63 & sitilouth & volume was \\
\hline 27 & t) & disheartered by & 64 & 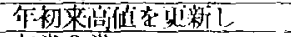 & hit a new year's high \\
\hline 28 & 八の期待加ら & on expectation of & 65 & 掌3 党 & ruling coalition \\
\hline 29 & GL口两 & edged up & 66 & 使安金 & Sumitomo Special Metals \\
\hline 30 & 八才方标 & high-tech shares & 67 & 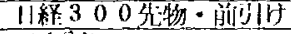 & Nikkei 300 futures Mng-cls: \\
\hline 31 & 栐子見x份 & wait-and-bee mood & 68 & $\langle$ XiIE & OSE \\
\hline 32 & 住林林 & Surnitomo Forestry & 69 & 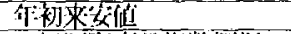 & year's low \\
\hline 33 & 11弗1動【交沙 & U.S. Japan auto talks & 10 & 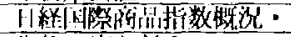 & Nikkei World Commodities: \\
\hline 34 & 物点 & "peculative buying of & 71 & 分物一住口付焉 & nikkei futures opg: \\
\hline 35 & 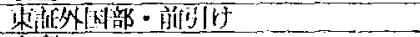 & Tokyo foreignatockn mng: & 72 & Hisj|b & morning close \\
\hline 36 & 玲利 & interestrates & 73 & 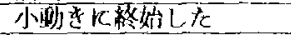 & inched up \\
\hline 37 & 11来11動 & the Japen-U.S.auto dispute & & & \\
\hline
\end{tabular}

Table 1: Samples of Fixed Collocations;

camnot be constructed compositionally. lor exam ple, No 9 means "lokyo Gold luture market ended trading for the day', but was never written as such. As well as No. 9, a number of sentence-level collocations were also extracted. No. 9, No. 18, No. 23, No, 26, No. 35, No. 56 and No. 67 are typical heads of the stock market report. These: expressions appear everyelay in stock market reports.

It is interesting to notice the varicty of fixed collocations. 'lhey differ in their constructions; nomn phrases, vert phrases, prepositional phases, and sentence-level. Although conventional methods focus on noun phrases or try to encompass all kinds of collocations at the same time, wo believe that fixed collocation is an importint class of collocation. It is useful to intensively study fixed collocations becanse the collocation of more complex structures is difficult to learn regardlessis of the method used.

Table 3 exemplifies the flexible collocations we acquired from the same corpus. No. 1 to No. 4 are typical expressions in stock market reports. 'These. collocation are extrencly useful for tomplate based machine translation systems. No. 5 is an example of a uscless collocation. Both Omron and Sumitomo Porestry ale company names that co-occur frequently in stock market reports, but these two companies have no dired relation. In fact, more than hall of all flexible collocations acquired were like No. 5. To remove useless collocit tions, const aants on the character types would be useful. Most useful Japanese flexible collocations contain at least one II imgana ${ }^{3}$ character. 'I'hus,

${ }^{3}$ Japanese has three types of characters (Hiragana, Katakana, and Kanji), each of which has different amonnte of information. In contrast, linglish has only 
many useless collocations can be removed by imposing this constraint on extracted strings.

It is also interesting to compare our results with a Japanese-English dictionary for economics (Iwatsu, 1990). About half of Table 4 and all of Table 3 are not listed in the dictionary. In particular, no verb-phrase or sentence-level collocations are not covered. These collocations are more useful for translators than noun phrase collocations, but greatly differ from domain to domain. Thus, it is difficult in general to hand-compile a dictionary that contains these kinds of collocations. Because our method automatically extracts these collocations, it will be of significant use in compiling domain specific dictionaries.

Finally, we briefly describe the coverage of the proposed method. For the corpus examined, $70 \%$ of the fixed collocations and $35 \%$ of the flexible collocations output by the method were correct. This level of performance was achieved in the face of two problems.

- The English text was not a literal translation. Parts of Japanese sentence were often omitted and sometimes appeared in a different English sentence.

- The data set was too small.

We are now constructing a larger volume of corpus to address the second problem.

\section{Conclusion}

We have described a new method for learning bilingual collocations from parallel corpora. Our method consists of two steps: (1) extracting useful word chunks by the word-level sorting technique and (2) constructing bilingual collocations by combining these chunks. This architecture reflects the fact that fixed collocations play a more crucial role than accepted in previous research. Our method not only extracts fixed collocations with high precision but also reduces the combinatorial explosion that would be otherwise considered inescapable in extracting flexible collocations. Although our research is in the preliminary stage and tested with a small number of Japanese stock market bulletins and their English, the experimental results have shown a number of interesting collocations that are not contained in a dictionary of economic terms.

\section{References}

Fric Brill. 1994. Some advances in transformation-based part of speech tagging. In Proc. 12th $A A A I$, pages 722-727.

Ido Dagan and Ken Church. 1994. Termight: identifying and translating technical terminol- ogy. In Proc. Fourth Conference on Applied Natural Language Processing, pages 34-40.

Pascale Fung. 1995. A pattern matching method for finding noun and proper noun translations from noisy parallel corpora. In Proc. $33 r d A C L$, pages $236-243$.

Gaston H. Gonnet, Ricardo A. Baeza-Yates, and Tim Snider, 1992. Information Retrieval, chapter 5, pages 66-82. Prentice-Hall.

Masahiko Haruno and Takefımi Yamazaki. 1996. High-Performance Bilingual Text Alignment Using Statistical and Dictionary Information. In Proc. 34th ACL.

Satoru Ikehara, Satoshi Shirai, and Hajime Uchino. 1996. A statistical method for extracting unitnerrupted and interrupted collocations from very large corpora. In Proc. COLING96.

Keisuke Iwatsu. 1990. TREND: Japanese-English Dictionary of Current Terms. Shougakkan.

Akira Kumano and Hideki Hirakawa. 1994. Building an MT dictionary from parallel texts based on linguisitic and statistical information. In Proc. 15th COLING, pages 76-81.

Julian Kupiec. 1993. An algorithm for finding noun phrase correspondences in bilingual corpora. In the 31st Annual Meeting of $A C L$, pages $17-22$

Sadao Kurohashi, Toshihisa Nakamura, Yuji Matsumoto, and Makoto Nagao. 1994. Improvements of Japanese morphological analyzer JUMAN. In Proc. International Workshop on Sharable Natural Language Resources, pages $22-28$.

Yuji Matsumoto, Hiroyuki Ishimoto, and Takehito Utsuro. 1993. Structural matching of parallel texts. In the 31 st $\Lambda$ nnual Meeting of $A C L$, pages 23-30.

Makoto Nagao and Shinsuke Mori. 1994, A new method of n-gram statistics for large number of $\mathrm{n}$ and automatic extraction of words and pharases from large text data of japanese,. In Proc. 15th COLING, pages 611-615.

Satoshi Sato and Makoto Nagao. 1990. Toward memory-based translation. In Proc. $131 \mathrm{~h} \mathrm{COL-}$ $I N G$, pages $247-252$.

Frank Smadja, Kathleen McKeown, and Vasileios Matzivassiloglou. 1996. Translating collocations for bilingual lexicons: A statistical approach. Computational Linguistics, 22(1):1-38, March.

Frank Smadja. 1993. Retricving collocations from text: Xtract. Computational Linguistics, 19(1):143-177, March.

one type of character. 